Pure and Applied Mathematics Quarterly

Volume 9, Number 2

(Special Issue: In honor of

Dennis Sullivan, Part 2 of 2)

$371-385,2013$

\title{
How Dennis and I Intersected
}

\author{
Jim Stasheff
}

\begin{abstract}
This paper is a slightly modified version of the talk I gave at the Dennisfest. After some reminiscences about how things were in the good old days, including how Dennis' work and mine were somewhat transverse ;-) I will review and update my ancient, unpublished (now arXived) paper with Mike Schlessinger on the deformation theory of rational homotopy types. Then, I will return briefly to another transverse intersection of my work with Dennis'.
\end{abstract}

Keywords: deformation theory, rational homotopy theory, classifying space, moduli space, $L_{\infty}$-structure.

\section{Contents}

1. Introduction 372

2. Manifolds? 373

3. A point of intersection - Poincaré duality spaces 373

4. Quillen's approach to rational homotopy theory 374

5. Work with Steve Halperin 375

6. Work with Mike Schlessinger 376

6.1. Classifying space versus moduli space 378

6.2. $L_{\infty}$-structure on $H(L) \quad 379$

Received November 4, 2011. 
7. Examples and computations 380

8. Extension to fibrations 381

8.1. Algebraic model of a fibration 381

8.2. Twisting cochains as perturbations 382

9. A string of intersections 383

$\begin{array}{ll}\text { References } & 383\end{array}$

\section{INTRODUCTION}

Dennis refers to me at times as Mr. $A_{\infty}$. Perhaps I should call him Mr. $E_{\infty}$ since his work has been so much more diverse than mine. Indeed, $E_{\infty}$ implies a lot more structure than $A_{\infty}$, but we have overlapped in small but significant ways.

One of the advantages of being of a certain age is that I am free to reminisce about how things were in the "good old days" - or at least how I remember them.

At one time, our interaction might be pictured

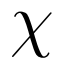

That intersection is primarily Dennis' minimalist approach to rational homotopy theory which, for some of us, is more accessible and hands on than Quillen's 'maximalist', that is, categorical. On the other hand, Quillen's version does highlight certain functors which were hidden for a while in Dennis'.

I mourn the tragic loss of Dan even as I rejoice in Dennis' presence.

One of the key aspects of his models of rational homotopy types, though overlooked at the time, was that his models were of the form of a free graded commutative algebra on the suspension of an $L_{\infty}$-coalgebra. The $L_{\infty}$ structure was in essence the differential on the algebra. 
Perhaps this structure or the problem of commutative cochains is what led to his interest in $\infty$-structures. Commutativity over the integers destroys associativity, but only up to homotopy, in fact strong homotopy, i.e. an $A_{\infty}$-structure. (In my talk, I said: "Maybe Dennis would care to set the record straight now, continuing his fine imitation of Gel'fand.", referring to his ability to take over the 'podium' of the seminar as called for.)

Some people refer to Sullivan models, meaning the minimal ones, but sometimes there are advantages to being not so efficient, as we will see.

\section{MANIFOLDS?}

That graphic above (the large $\chi$ ) is a deliberate double entendre. Not only are our interests transverse, but I have never used manifold structures.

What never?

Well, hardly ever.

Of course the "Characteristic Classes" book with Milnor starts off with a treatment of manifolds, but that part Milnor wrote years after the original lectures, for which I was just the scribe - a very fortunate scribe.

In "Differential homological algebra and homogeneous spaces," with Dale Husemoller and John Moore [13], the emphasis was on the first topic, though motivated by the example of the second.

One place where I really was interested in manifolds was "Manifolds of the homotopy type of (non-Lie) groups" [28], which I owe in part to Dennis in his 1970 MIT notes [30] for the idea of breaking apart a space into its p-primary components and to Zabrodsky [33] for the idea of mixing together p-primary components from different spaces.

\section{A Point of intersection - Poincaré DUality sPaCes}

One paper I wrote was devoted to rational Poincaré duality spaces [27], motivated in part by a duality at the level of Massey products.

My favorite example is

$$
S p(5) / S U(5) \text {. }
$$


Its cohomology algebra is isomorphic to that of

$$
S^{6} \times S^{25} \# S^{10} \times S^{21}
$$

where \# denotes connected sum. But that space has no non-trivial Massey products, while $S p(5) / S U(5)$ has two non-trivial 3-fold Massey products $<, \quad,>$ (as follows from computations by Borel [2]). If we denote the algebra generators by their dimensions, then $\langle 6,6,10\rangle=21$ and $\langle 6,10,10\rangle=25$. The duality also exhibits the fundamental slide relation [18]:

$$
<6,6,10>10=6<6,10,10>
$$

There is no homotopy type with this cohomology algebra and only one non-trivial Massey product.

Recently Larry Taylor [31] has found a much better way to exhibit the duality at the level of Massey products with clever control of the indeterminacy. The chief idea is to convert the Massey triple product $\langle x, y, z\rangle$ to a linear operation $\langle x, ?, z\rangle$, defined for all $y$ such that $x y=0=y z$ by fixing $x$ and $z$. The usual slide relation

$$
w<x, y, z>= \pm<w, x, y>z
$$

then gives an explanation for the pairs of Massey product in the above example.

\section{QUiLlen'S APPROACH TO RATIONAL HOMOTOPY THEORY}

Quillen emphasized differential graded Lie algebras (dgLs) as well as differential graded commutative coalgebras (dgccs): for a simply connected space $X$, there is a $\operatorname{dgL} L_{X}$ such that $H\left(L_{X}\right)$ is isomorphic as graded Lie algebra to the rational homotopy groups of the loop space of $X$ with respect to the Samelson product. Baues and Lemaire [1] gave a construction of $L_{X}$ as a free graded Lie algebra on the despsupension of the reduced homology of $X$. Hain [7] studied this construction from the point of view of Chen's connections. Simple connectivity in the dg algebra setting mean $A^{0}=\mathbf{Q}$ and $A^{1}=0$.

Let $\mathcal{L}$ denote the free graded Lie algebra functor on the suspension of a graded vector space. For a simply connected rational Poincaré duality space $Y$ with

fundamental class $\mu \in H^{N}$, choose a self-dual basis $\left\{x_{1}, \ldots, x_{q}, x^{q}, \ldots, x^{1}\right\}$ for degrees $k: 0<k<N$, so that $x_{i} x^{i}=\mu$. I was able to show: 
Theorem 1. [27] There is a differential graded Lie algebra model $\mathcal{L}(H(Y))$ with

$$
d(\mu)=1 / 2 \Sigma\left[x_{i}, x^{i}\right]
$$

Equivalently $Y=X \cup e^{N}$ where $e^{N}$ is attached by ordinary Whitehead products (not iterated) with respect to some basis of the rational homotopy groups of $X$.

\section{Work With Steve Halperin}

Disclaimer The results here are stated in the hopes of conceptual accessibility, not in maximal generality; i.e. being simply connected can often be generalized to being nilpotent.

Sullivan models sometimes refer to minimal ones, but Halperin and I [8] made good use of perturbing the minimal model of a formal space to a non-minimal model of a general simply connected rational space (henceforth, for the rest of the paper, called space).

Let $\mathcal{H}$ denote a graded algebra such that for some space or $\operatorname{dgca}\left(A, d_{A}\right)$ we have an isomorphism $\phi: \mathcal{H} \rightarrow H\left(\left(A, d_{A}\right)\right)$. Given this, we constructed a canonically filtered non-minimal Sullivan model for $\left(A, d_{A}\right)$ by perturbing the minimal model for the graded algebra $\mathcal{H}$. (Specification of $\phi$ plays a role in the classification to come.) In fact, the minimal model for $\mathcal{H}$ is a purely algebraic construct, closely related to a multiplicative resolution of $\mathcal{H}$ by free commutative algebras, sometimes called the Koszul-Tate resolution (and extended to the graded case by Jozefiak) $[25,24]$. The filtration corresponds to the resolution degree.

We refer to the total degree minus resolution degree as the weight.

By a perturbed differential $D$, we mean a derivation that can be written as $D=d+p$ where $p$ lowers weight by at least 2 . That is, $D=d+d_{2}+d_{3}+\cdots$, where $d_{i}$ lowers weight by $i$.

A perturbation could also be called a deformation. Conversely, deformations can often be studied as perturbed differentials.

If $S$ and $T$ are simply connected (more generally, rationally nilpotent) spaces and $f: H^{*}(S ; \mathbf{Q}) \rightarrow H^{*}(T ; \mathbf{Q})$ is an isomorphism of algebras, we used the filtered models to construct a sequence of classes, $O_{n}(f)$, to be called obstructions. 
Theorem 2. [8] The isomorphism $f$ can be realized by a rational homotopy equivalence if and only if the obstructions $O_{n}(f)$ all vanish.

The next obvious question was "how different can rational homotopy types be if the cohomology algebras agree?".

For example, Theorem 2 is one of many ways that it can be seen that:

Theorem 3. [5, 22, 8] For $n>1$ and $H^{i}=0$ for $i<n$ and for $i \geq 3 n-1$, then $H$ is intrinsically formal, i.e., there is only one homotopy type with the given cohomology algebra.

For rational Poincaré duality spaces, the hypothesis can be extended to $H^{i}=0$ for $i<n$ and for $i \geq 4 n-1$.

We gave several other illustrative examples with specific computations, but not a general answer, except to remark that our procedure identifies the set of homotopy types with given rational cohomology algebra $\mathcal{H}$ in terms of the set of homology preserving deformations of the minimal model of $(\mathcal{H}, 0)$.

\section{Work With Mike Schlessinger}

Sometime after my work with Halperin, Mike Schlessinger and I [25, 24] made contact mathematically - geographics were not a problem; our offices at UNC were across the hall from each other. As I recall, it happened like this:

Something led us to compare notes on the Koszul-Tate resolution of

$$
\mathbf{Q}\left[x_{1}, x_{2}\right] / x_{i} x_{j}=0,
$$

which Mike regarded as pure algebra and I regarded as the cohomology of $S^{2} \vee S^{2}$. Similarly, Mike spoke the language of classical deformation theory while I used that of homological perturbation theory. Fortunately, we each learned to speak the other's language. (In contrast, it took decades for Murray Gerstenhaber and me to realize we had common techniques, though our breakthrough papers $[6,26]$ had each appeared in 1963 - it was a very good year.)

It was from Mike that I learned the yoga: deformation problems are controlled by dg Lie algebras. Later this yoga was extended to control by $L_{\infty}$-algebras so as to include the appropriate $L_{\infty}$-uniqueness. 
In order to classify homotopy types, we first chose to use a particular nonminimal Sullivan model of the formal space with the given cohomology algebra $\mathcal{H}$, using the adjointness between dgcas and dg Lie coalgebras.

Quillen's approach to rational homotopy theory is at the chain rather than cochain level. In [23], he constructs a functor from simply connected rational spaces to dg Lie algebras and then applies a generalized Chevalley-Eilenberg construction $\mathcal{C}$ to obtain a dgc coalgebra model. (The functor $\mathcal{C}$ also appears in work of J. C. Moore [20] the following year, I believe independently.) The functor $\mathcal{A}(\quad)=\operatorname{Hom}(\mathcal{C}(\quad), \mathbf{Q})$ from dg Lie algebras to dga's fits more readily into a traditional cohomological exposition, but the usual subtleties of the Hom functor necessitate the detour into the more natural differential graded coalgebras. We will still need to have recourse to $\hat{\mathcal{C}}$, the completion of $\mathcal{C}$ with respect to a suitable filtration.

Apply the cofree graded Lie coalgebra $\mathcal{L}^{c}$ to the shifted augmentation ideal of $\mathcal{H}$, with the differential $d_{\mathcal{L}^{c}}$ defined by extending the multiplication in $\mathcal{H}$ as a coderivation (after shifting). Then form the free graded commutative algebra $\mathcal{A}\left(s \mathcal{L}^{c} \mathcal{H}\right)$ with differential $d$ determined by $d_{\mathcal{L}^{c}}$ and the cobracket. This construction defines a rational dgca $A$ which is manifestly formal. For a general rational space/dgca A, the argument in my work with Halperin can be used to perturb $d_{\mathcal{A}\left(s \mathcal{L}^{c} s \mathcal{H}\right)}$ to give a model for $\left(A, d_{A}\right)$.

Although perturbations are always of total degree 1, they sit naturally in a subdg Lie algebra of $\operatorname{Der}\left(\mathcal{A}\left(s \mathcal{L}^{c} \mathcal{H}\right)\right)$. We will also have need for perturbations of the differential of $\mathcal{L}^{c} s \mathcal{H}$, which sit naturally in a sub-dg Lie algebra of $\operatorname{Coder}\left(\mathcal{L}^{c} s \mathcal{H}\right)$. These we formalize with a definition.

Definition 4. Denote by Pert $\mathcal{A}\left(s \mathcal{L}^{c} s \mathcal{H}\right)$ the $d g$ Lie algebra of weight decreasing derivations of $\mathcal{A}\left(s \mathcal{L}^{c} s \mathcal{H}\right)$. Similarly denote by $\operatorname{Pert}\left(\mathcal{L}^{c} s \mathcal{H}\right)$ the dg Lie algebra of weight decreasing coderivations of $\mathcal{L}^{c} \mathcal{H}$, where a perturbation of $\mathcal{L}^{c} s \mathcal{H}$ is a Lie coderivation $p$ of the same degree as $d$ such that $p$ increases cobracket length by at least 2 and $(d+p)^{2}=0$.

Theorem 5. Let $\mathcal{H}$ be a simply connected graded commutative algebra of finite type. The set of augmented homotopy types of dgca's

$$
(A, \phi: \mathcal{H} \approx H(A))
$$

is in 1-1 correspondence with the path components of $\hat{\mathcal{C}}\left(\operatorname{Pert} \mathcal{A}\left(s \mathcal{L}^{c} s \mathcal{H}\right)\right)$. 
A graded coalgebra map from the rationals $\mathbf{Q}$ as a coalgebra serves as a point and a path is an appropriate kind of homotopy between points, using an 'interval' dgc coalgebra. (There is indeed some subtlety there [24].)

The easy part of the theorem is the observation as in [8] that two perturbed models have the same augmented homotopy type provided they are related by an automorphism (of the underlying gca) of the form: Id plus "terms which decrease weight".

The hard part is to show that a homotopy gives rise to such an equivalence.

Now $\mathcal{A}\left(s \mathcal{L}^{c} s \mathcal{H}\right)$ is rather large, so we are interested in comparing perturbations of $\mathcal{L}^{c} s \mathcal{H}$ with the corresponding induced perturbations of $\mathcal{A}\left(s \mathcal{L}^{c} s \mathcal{H}\right)$, for the freeness of the latter implies that a perturbation of $\mathcal{L}^{c}(s \mathcal{H})$ generates one in $\mathcal{A}\left(\mathcal{L}^{c}(s \mathcal{H})\right)$ with respect to the induced filtration.

Theorem 6. For simply connected $\mathcal{H}$ of finite type, the natural dg Lie algebra map $\left.\operatorname{Pert}\left(\mathcal{L}^{c} s \mathcal{H}\right)\right) \rightarrow \operatorname{Pert} \mathcal{A}\left(s \mathcal{L}^{c} s \mathcal{H}\right)$ is a homology isomorphism.

6.1. Classifying space versus moduli space. Theorem 5 indicates $\hat{\mathcal{C}}\left(\operatorname{Pert} \mathcal{A}\left(s \mathcal{L}^{c}(\mathcal{H})\right)\right)$ is rather like a model of a classifying space, an idea we will reinforce later. More to the point right now, the set of path components can be regarded as a topological space, but then we need to make a further identification by the action of Aut $\mathcal{H}$, the group of automorphisms of $\mathcal{H}$, though the quotient can fail to be even Hausdorff.

On the other hand, since perturbations satisfy the defining equation (equivalent to the Maurer-Cartan equation), we can change point of view to that of algebraic geometry: varieties and moduli spaces.

Definition 7. For any $d g$ Lie algebra L, we define the incorporated $d g$ Lie algebra $(L[d], a d d)$ by adjoining a single new generator also called $d$ with the obvious relations. The variety $V_{L} \subset L[d]$ is defined as

$$
\left\{p \in L^{1} \mid(d+p)^{2}=0\right\} .
$$

If $L$ is complete with respect to the $L^{0}$ filtration, the gauge action is

$$
p \mapsto(\exp b)(d+p)-d \quad \text { for } b \in L^{0} .
$$

Neither the variety $V_{L}$ nor the gauge action is uniquely determined by the problem, but the quotient by the gauge action is (up to appropriate isomorphism). 
Implicit in the use of quasi-isomorphisms, even for strict dg Lie algebras, is the fact that $L_{\infty}$-morphisms respect the deformation and moduli space functors.

For the classification problem, we want $V_{L}$ for $\left.L=\operatorname{Pert}\left(\mathcal{L}^{c} s \mathcal{H}\right)\right)$. A simple example is $\mathcal{H}=H\left(S^{2} \vee S^{2} \vee S^{5}\right)[8] \S 6.6$. Let the generators be $x_{2}, y_{2}, z_{5}$. For dimensional reasons $V_{L}$ is $\mathbf{Q}^{2}$. Finally, $A u t \mathcal{H}=G L(2) \times G L(1)$ acts on $H^{1}(L)$ so as to give two orbits: $(0,0)$ and the rest. The space of rational homotopy types is

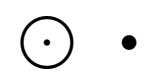

meaning the non-Hausdorff two-point space with one open point and one closed. We can also represent this as

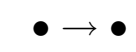

meaning one orbit is a limit point of the other.

6.2. $L_{\infty}$-structure on $H(L)$. Since $\operatorname{Pert}\left(\mathcal{L}^{c}(\mathcal{H})\right)$ is still rather large for effective computation, it is helpful to have at hand the $L_{\infty}$-structure induced on $H(L)$ for any dg Lie algebra $L$. Even though $H(L)$ inherits a strict dgLie structure (with $d=0)$, there is in general a highly non-trivial $L_{\infty}$-structure such that $L$ and $H(L)$ are equivalent as $L_{\infty}$ algebras. This transfer of structure began in the case of $\mathrm{dg}$ associative algebras with the work of Kadeishvili [14]. The definitive treatment in the Lie case (which is more subtle) is due to Huebschmann [11, 12].

As we have recalled, the structure of a dg Lie algebra is captured by the Chevalley-Eilenberg complex $\mathcal{C}(s L)$. We can construct a minimal model of $\mathcal{C}(s L)$ which is a perturbation of $\mathcal{C}(s H(L))$, i.e.

$$
d=0+[,]_{H(L)}+\text { terms of bracket length }>2 .
$$

These higher order terms correspond precisely to the higher order brackets of an $L_{\infty}$-algebra structure on $H(L)$.

In the case in which the dg Lie algebra is $\operatorname{Pert}\left(\mathcal{L}^{c}(\mathcal{H})\right)$, some of these higher order terms can be related to Massey products and attaching maps (see the next section). Although Massey products suffer from having restricted domains and considerable indeterminacy, when defined, they represent the higher order brackets on $H\left(\operatorname{Pert}\left(\mathcal{L}^{c}(\mathcal{H})\right)\right.$, which are better defined. 


\section{ExAmples AND COMPUTATIONS}

There are some easy consequences for the classification from conditions on $H\left(\operatorname{Pert}\left(\mathcal{L}^{c}(\mathcal{H})\right)\right)$ which follow the route of classical deformation theory: results that depend on vanishing of $H\left(\operatorname{Pert}\left(\mathcal{L}^{c}(\mathcal{H})\right)\right)$ in low degrees. Let's turn now to computations of manageable size.

$\operatorname{Pert}\left(\mathcal{L}^{c}(\mathcal{H})\right)$ can be identified with a subspace of $\operatorname{Hom}\left(\mathcal{L}^{c}(\mathcal{H}), \mathcal{H}\right)$ and hence each perturbation as a sum of elements of $\operatorname{Hom}\left(\overline{\mathcal{H}}^{\otimes k+2}, \mathcal{H}\right)$ which lower the internal $\mathcal{H}$-degree by $k$. This permits an interpretation in terms of attaching maps, for example, in case the formal space is a wedge of spheres

$$
X=\bigvee S^{n_{i}}
$$

The dual of the algebra of rational homotopy groups $\pi_{*}(\Omega X) \otimes \mathbf{Q}$ is then isomorphic to $\mathcal{L}^{c}(H(X))$.

Recall that attaching a cell by an ordinary Whitehead product $\left[S^{p}, S^{q}\right]$ means the cell carries the product cohomology class. Massey (and Uehara) $[32,19]$ introduced Massey products in order to detect cells attached by iterated Whitehead products such as $\left[S^{p},\left[S^{q}, S^{r}\right]\right]$.

The Massey product interpretation is particularly helpful when the perturbation consists of only one term $\theta_{k}$, if we identify such a perturbation $\theta_{k}$ with a homomorphism $\theta_{k}: H^{\otimes k+2} \rightarrow H$,

Adopting the language of deformation theory, we refer to a candidate perturbation $\theta$ as an infinitesimal deformation. If $(d+\theta)^{2} \neq 0$, we say $\theta$ is obstructed.

One of the simplest examples of this phenomenon is $X=S^{3} \vee S^{3} \vee S^{8} \vee S^{13}$. We use a dual notation and write:

$$
\theta=\left[x_{1},\left[x_{1}, x_{2}\right]\right] \partial x_{8}+\left[x_{2},\left[x_{1}, x_{8}\right]\right] \partial x_{13},
$$

then $[\theta, \theta]$ is not cohomologous to 0 .

In terms of cells, this means we cannot attach simultaneously both $e^{8}$ to realize $\left\langle x_{1}, x_{1}, x_{2}\right\rangle$ and attach $e^{13}$ to realize $\left\langle x_{2}, x_{1}, x_{8}\right\rangle$.

The first example of "continuous moduli", i.e., of a one-parameter family of homotopy types, was first mentioned to us by John Morgan (cf. [21, 5]). Let $H=H\left(S^{3} \vee S^{3} \vee S^{12}\right)$, so that the attaching map $\alpha$ is in $\pi_{11}\left(S^{3} \vee S^{3}\right) \otimes \mathbf{Q}$ which is of dimension 6, while Aut $H=G L(2) \times G L(1)$ is of dimension 5. Alternatively, the 
space of 5 -fold Massey products $H^{\otimes 5} \rightarrow H$ is of dimension 6 and so distinguishes at least a 1- parameter family.

\section{Extension to FIBRATiOnS}

The construction of a rational homotopy model for a classifying space for fibrations with given fibre was sketched briefly by Sullivan [29]. The classification of rational homotopy types by homotopy classes of $\mathrm{dg}$ coalgebra maps of $\mathbf{Q} \rightarrow$ $\hat{C}(\mathcal{L})$ generalizes naturally to classification of rational homotopy fibrations by maps of the base dgcc to $\hat{C}(\mathcal{L})$ where $\mathcal{L}$ is the dg Lie coalgebra corresponding to self homotopy equivalences of the fibre $F$.

8.1. Algebraic model of a fibration. For simplicity of exposition, let's assume enough conditions so we can deal with the algebraic model of a fibration as a twisted tensor product. Consider topological fibrations, i.e., maps of spaces

$$
\mathcal{F} \rightarrow \mathcal{E} \stackrel{p}{\rightarrow} \mathcal{B}
$$

such that $p^{-1}(*)=\mathcal{F}$ and $p$ satisfies the homotopy lifting property. We have not only the corresponding maps of generalized differentil form cgda's

$$
A^{*}(\mathcal{B}) \rightarrow A^{*}(\mathcal{E}) \rightarrow A^{*}(\mathcal{F})
$$

but $A^{*}(\mathcal{E})$ is an $A^{*}(\mathcal{B})$-algebra and, under reasonable assumptions, there is an $A^{*}(\mathcal{B})$-derivation $D$ on $A^{*}(\mathcal{B}) \otimes A^{*}(\mathcal{F})$ and an equivalence of cdgas between

$$
A^{*}(\mathcal{E}) \text { and }\left(A^{*}(\mathcal{B}) \otimes A^{*}(\mathcal{F}), D\right) .
$$

By a sleight of hand, let us rename these $\operatorname{dgcas} F, E$ and $B$. Both the algebra structure and the differential may be twisted, but if we assume that $F$ is free as a cga, e.g. a model for $F$, then it follows that $E$ is strongly equivalent to

$$
B \stackrel{i}{\longrightarrow} B \otimes F \stackrel{p}{\longrightarrow} F
$$

with the $\otimes$-algebra structure. The differential in $E=B \otimes F$ then has the form

$$
d_{\otimes}+\tau
$$

where

$$
d_{\otimes}=d_{B} \otimes+1 \otimes d_{F}
$$


Let $\bar{B}$ denote the augmentation ideal of $B$. The "twisting term" $\tau$ lies in the sub-dg Lie algebra of $\operatorname{Der}(B \otimes F)$ consisting of those derivations of $B \otimes F$ which vanish on $B$ and reduce to 0 on $F$ via the augmentation.

8.2. Twisting cochains as perturbations. Assuming $B$ is connected, $\tau$ does not increase the $F$-degree so we regard $\tau$ as a perturbation of $d_{\otimes}$ on $B \otimes F$ with respect to the filtration by $F$ degree. The twisting term must satisfy the integrability conditions (aka the Maurer-Cartan equation):

$$
(d+\tau)^{2}=0 \text { or }[d, \tau]+\frac{1}{2}[\tau, \tau]=0 .
$$

To obtain strong equivalence classes of fibrations, we pass to the quotient by the action of automorphisms $\theta$ of $B \otimes F$ which are the identity on $B$ and reduce to the identity on $F$ via augmentation. Assuming $B$ is connected, then $\theta-1$ must take $F$ to $\bar{B} \otimes F$ and therefore lowers the degree in $F$, so that $\phi=\log \theta=\log (1+(\theta-1))$ exists; thus $\theta=\exp (\phi)$ for some $\phi$ in $\operatorname{Der}^{0}(F, B \otimes F)$.

If we set $\mathcal{L}(B, F)=\operatorname{Der}(F, \bar{B} \otimes F)$, then for $B$ connected, we may repeat much of what we did for the case $B=\mathbf{Q}$. Dualizing with impunity, we consider

$$
\mathcal{A}(s \mathcal{L}(B, F)) .
$$

Theorem 8. For connected $B$ and reasonable $F$, free of finite type, the set of strong fibre homtopy equivalence classes of fibrations

$$
B \rightarrow E \rightarrow F
$$

is in bijection with the set of homotopy classes of maps $\mathcal{A}(s \mathcal{L}(B, F)) \rightarrow B$.

Thus $\mathcal{A}(s \mathcal{L}(B, F))$ is very much like a model for the classifying space. The problem is that it has terms of negative degree, so, presto chango, we truncate it appropriately.

Mike, of course, went further and provided the moduli space specification.

Theorem 9. [24] For fixed $\mathcal{H}$, the set of homotopy types of pairs $(X, \phi: \mathcal{H} \simeq$ $H(X))$ can be represented as the quotient $V / G$ of a (perhaps infinite dimensional) conical rational algebraic variety $V$ modulo a pro-unipotent (algebraic) group action $G$. 


\section{A STRING OF INTERSECTIONS}

Dennis and I have each dealt with homotopy theory arising from strings. For Dennis, there is his work with Chas [3] which gave rise to a major industry of string topology, whereas in my work with Kajiura [15], [17],[16] and in work of Hoefel [10], [9], there is translation of the physicists string field theory into homotopical algebra. Earlier, I had planned to compare and contrast the various $\infty$-algebra structures that arise in these different approaches, but I find that there are now established a plethora of such structures and considerable further work in progress, e.g. the minimal model of $B V_{\infty}$-structures of Drummond-Cole and Vallette [4].

Of course, Dennis and his progeny work in the context of manifolds or at least Poincaré Duality, while I impose no such constraint. A more important difference is that string topology is developed as a homology or even a chain level structure, whereas string field theory is contravariant, in terms of functions or forms on a space of strings.

\section{REFERENCES}

[1] H. J. Baues and J.-M. Lemaire. Minimal models in homtopy theory. Math. Ann. 225, pages 219-242, 1977.

[2] A. Borel. Sur la cohomologie des espaces fibrés principaux et des espaces homogenes de groupes de Lie compacts. Annals of Math., 57:115-207, 1953.

[3] M. Chas and D. Sullivan. Closed string operators in topology leading to Lie bialgebras and higher string algebra. In The legacy of Niels Henrik Abel, pages 771-784. Springer, Berlin, 2004.

[4] G. C. Drummond-Cole and B. Vallette. The minimal model for the Batalin-Vilkovisky operad. Selecta Mathematica, 2012. arXiv:math1105.2008.

[5] Y. Félix. Dénombrement des types de k-homotopie. Théorie de la déformation. Bulletin. Soc. Math. France, 108(3), 1980.

[6] M. Gerstenhaber. The cohomology structure of an associative ring. Ann. Math., 78:267-288, 1963.

[7] Richard M. Hain. Twisting cochains and duality between minimal algebras and minimal Lie algebras. Trans. Amer. Math. Soc., 277(1):397-411, 1983.

[8] S. Halperin and J. Stasheff. Obstructions to homotopy equivalences. Advances in Math., 32:233-279, 1979.

[9] E. Hoefel. OCHA and the swiss-cheese operad. J. Homotopy Relat. Struct., 4(1):123-151, 2009. 
[10] E. Hoefel. On the coalgebra description of OCHA. J. Pure Appl. Algebra, 216(3):734-741, 2012.

[11] J. Huebschmann. The Lie algebra perturbation lemma. In Higher structures in geometry and physics, volume 287 of Progr. Math., pages 159-179. Birkhäuser/Springer, New York, 2011.

[12] J. Huebschmann. The sh-Lie algebra perturbation lemma. Forum Math., 23(4):669-691, 2011.

[13] D. Husemoller, J. C. Moore, and J. Stasheff. Differential homological algebra and homogeneous spaces. J. Pure Appl. Algebra, 5:113-185, 1974.

[14] T.V. Kadeishvili. On the homology theory of fibre spaces. Russian Math. Surv., 35:3:231238, 1980. math.AT/0504437.

[15] H. Kajiura and J. Stasheff. Homotopy algebras inspired by classical open-closed string field theory. Comm. Math. Phys., 263(3):553-581, 2006.

[16] H. Kajiura and J. Stasheff. Open-closed homotopy algebra in mathematical physics. $J$. Math. Phys., 47(2):023506, 28, 2006.

[17] H. Kajiura and J. Stasheff. Homotopy algebras of open-closed strings. In Groups, homotopy and configuration spaces, volume 13 of Geom. Topol. Monogr., pages 229-259. Geom. Topol. Publ., Coventry, 2008.

[18] D. Kraines. Massey higher products. Trans. Amer. Math. Soc., 124:431-449, 1966.

[19] W.S. Massey. Some higher order cohomology operations. In Symposium Internacional de Topologia Algebraica, pages 145-154, 1958.

[20] J. C. Moore. Differential homological algebra. In Actes du Congrès International des Mathématiciens (Nice, 1970), volume Tome 1, pages 335-339. Gauthier-Villars, Paris, 1971.

[21] J. Neisendorfer. Lie algebras, coalgebras and rational homotopy theory of nilpotent spaces. Pac. J. M., 74:429-460, 1978.

[22] J. Neisendorfer and T. Miller. Formal and coformal spaces. Illinois Journ. Math., 22(4):565$580,1978$.

[23] D. Quillen. Rational homotopy theory. Annals of Mathematics, 90:205-295, 1969.

[24] M. Schlessinger and J. Stasheff. Deformation theory and rational homotopy type. arXiv: 1211.1647.

[25] M. Schlessinger and J. Stasheff. Rational homotopy theory - obstructions and deformations. In Proc. Conf. on Algebraic Topology, Vancouver, pages 7-31, 1977. LMM 673.

[26] J. Stasheff. Homotopy associativity of H-spaces, II. Trans. Amer. Math. Soc., 108:313-327, 1963.

[27] J. Stasheff. Rational Poincaré duality spaces. Illinois Journ. Math., 27:104-109, 1983.

[28] J. D. Stasheff. Manifolds of the homotopy type of (non-Lie) groups. Bull. Amer. Math. Soc., 75:998-1000, 1969.

[29] D. Sullivan. Infinitesimal computations in topology. Pub. Math. IHES, pages 269-331, 1977.

[30] D. P. Sullivan. Geometric topology: localization, periodicity and Galois symmetry, volume 8 of K-Monographs in Mathematics. Springer, Dordrecht, 2005. The 1970 MIT notes, Edited and with a preface by Andrew Ranicki. 
[31] L. R. Taylor. Controlling indeterminacy in Massey triple products. Geom. Dedicata, 148:371-389, 2010.

[32] H. Uehara and W.S. Massey. The Jacobi identity for Whitehead products. In Algebraic Geometry and Topology, A Symposium in Honor of S. Lefschetz, pages 361-377, 1957.

[33] A. Zabrodsky. Homotopy associativity and finite CW complexes. Topology, 9:121-128, 1970.

Jim Stasheff

Mathematics Department

University of North Carolina at Chapel Hill and University of Pennsylvania

E-mail: jds@math.upenn.edu 